\title{
Stem Cell Therapy for Retinal Degeneration: The Evidence to Date
}

\section{Amit Sharma (iD \\ Bithiah Grace Jaganathan}

Stem Cells and Cancer Biology Research Group, Department of Biosciences and Bioengineering, Indian Institute of Technology Guwahati, Guwahati, Assam, 781039, India
Correspondence: Bithiah Grace Jaganathan Stem Cells and Cancer Biology Research Group, Department of Biosciences and Bioengineering, Indian Institute of Technology Guwahati, Guwahati, Assam, 781039, India

Tel +91 36I 2582219

Email bithiahgj@iitg.ac.in
Abstract: There is a rise in the number of people who have vision loss due to retinal diseases, and conventional therapies for treating retinal degeneration fail to repair and regenerate the damaged retina. Several studies in animal models and human trials have explored the use of stem cells to repair the retinal tissue to improve visual acuity. In addition to the treatment of age-related macular degeneration (AMD) and diabetic retinopathy (DR), stem cell therapies were used to treat genetic diseases such as retinitis pigmentosa (RP) and Stargardt's disease, characterized by gradual loss of photoreceptor cells in the retina. Transplantation of retinal pigment epithelial (RPE) cells derived from embryonic stem cells (ESCs) and induced pluripotent stem cells (iPSCs) have shown promising results in improving retinal function in various preclinical models of retinal degeneration and clinical studies without any severe side effects. Mesenchymal stem cells (MSCs) were utilized to treat optic neuropathy, RP, DR, and glaucoma with positive clinical outcomes. This review summarizes the preclinical and clinical evidence of stem cell therapy and current limitations in utilizing stem cells for retinal degeneration.

Keywords: retinal degeneration, retinal pigment epithelial cells, mesenchymal stem cells, embryonic stem cells, induced pluripotent stem cells, retinitis pigmentosa

\section{Introduction}

Retinal degeneration is one of the major reasons for vision loss, and stem cell therapy has been extensively investigated to repair and regenerate damaged retinal cells. Several types of stem cells have been tested in preclinical and clinical trials to understand their efficiency in reversing retinal degeneration. To date, human embryonic stem cells (hESCs)-, induced pluripotent stem cells (iPSCs)-derived RPE cells, mesenchymal stem cells (MSCs) and retinal progenitor cells (RPCs) have been tested in addition to paracrine factors and exosomes derived from MSCs.

Conventional therapies for retinal diseases slow the progression of the diseases; however, the long-term benefit is achieved by repairing and regenerating the damaged retinal tissue. Moreover, since the retina does not have intrinsic regenerative properties, stem cell therapies have been sought to repair and regenerate the damaged retina. ${ }^{1}$ Several preclinical and clinical studies have demonstrated that transplantation of stem cells and factors derived from stem cells produce clinically measurable improvement. This review will discuss the different stem cells utilized to treat retinal diseases and the clinical benefits and challenges in utilizing stem cells to treat retinal degeneration.

The etiology of retinal degenerative diseases includes genetic and non-genetic factors leading to the loss of photoreceptor cells and eventually the RPE cells. Agerelated macular degeneration (AMD) is one of the most common forms of vision loss, 


\section{Graphical Abstract}

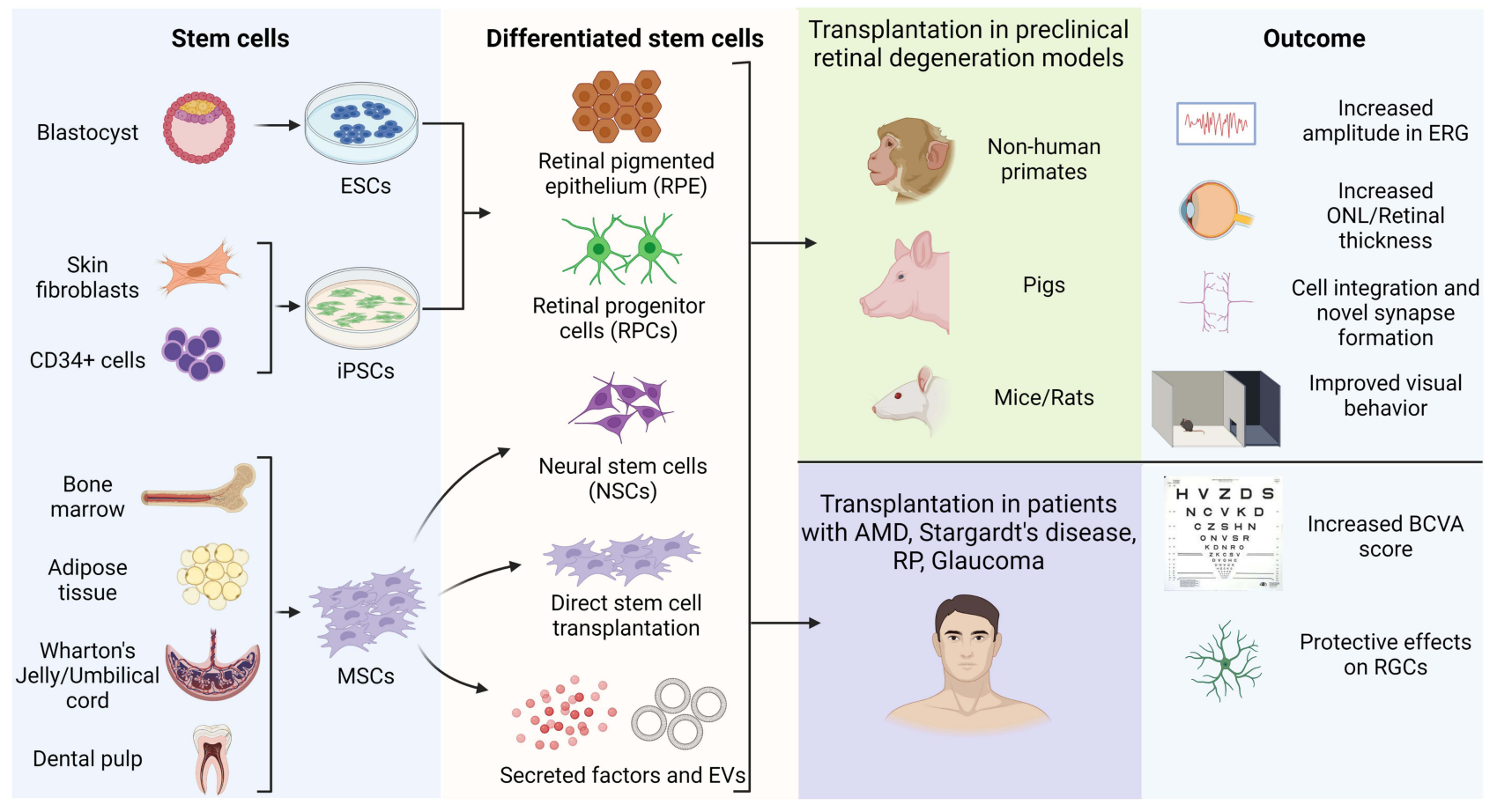

which might either be due to degradation of RPE cells (dry AMD) or choroidal neovascularization (wet AMD). Wet AMD is treated with anti-VEGF therapy, which can lead to clinical improvement in vision. ${ }^{2,3}$ In contrast, fewer therapeutic options are available to improve the vision in patients with dry AMD. Retinitis pigmentosa (RP) occurs due to autosomal $^{4}$ or X-linked mutations, which contribute to the degeneration of photoreceptors leading to vision loss. FDAapproved therapeutic interventions for RP include gene therapy for patients with a biallelic mutation in RPE65 ${ }^{5}$ and retinal prosthesis for late-stage RP. ${ }^{6}$ Diabetic retinopathy (DR) is caused due to chronic hyperglycemia and is treated with anti-VEGF to limit the neovascularization at the proliferative stage or late-stage DR. ${ }^{7}$ Although these conventional therapies have improved the disease prognosis, repeated administration is required to diminish the disease progression, and gene therapy is applicable only for those patients with vision loss due to specific mutations.

\section{Stem Cells for Retinal Diseases}

Stem cells were tested in several clinical trials, and the approaches include transplantation of undifferentiated stem cells, pre-differentiated stem cells, or stem cellderived factors. Several studies and clinical trials have utilized RPE cells derived from hESCs or iPSCs, and MSCs derived from various tissue sources and tested their retinal regenerative potential. Here, we have summarized and analyzed the potential of each cell type for the treatment of retinal disorders.

\section{Preclinical Studies with Stem Cells ESCs}

ESCs, due to their extensive proliferative and differentiation potential, have been used as a cell source to treat various degenerative diseases, including retinal degeneration. Subretinal transplantation of hESC-derived RPE cells in a preclinical mouse model of AMD showed no tumor growth with the transplanted cells detected at the injection site seven months after injection, ${ }^{8}$ and some injected cells formed an RPE monolayer above the native layer. ${ }^{9}$ In a similar study, RPCs derived from hESCs integrated into the mouse ganglion cell layer (GCL), expressed retinal ganglion cells (RGCs) marker Brn3a, ${ }^{10}$ and outer nuclear layer (ONL) thickness increased in the injected animals. ${ }^{11}$ In a study involving non-human primates, subretinal transplantation of hESC-derived retinal organoids was well tolerated and the transplanted cells integrated 
into the retinal layer in the injury site created by laser ablation. $^{12}$

\section{iPSCs}

iPSCs, similar to ESCs, have pluripotent differentiation ability but without ethical concerns. The human iPSC-derived retina was transplanted into the subretinal space of monkeys with laser-induced retinal injury and immune-deficient rats with RP. The transplanted cells integrated into the rat retina and formed synaptic connections with the host bipolar cells. In the monkey model, the transplanted cells integrated into the host retinal layer and improved electroretinogram (ERG) and visual guided saccade (VGS) scores were observed. ${ }^{13}$ Similarly, in an RP mouse model, subretinal transplantation of iPSCderived RPE spheroids delayed thinning of retinal ONL, increased pigment epithelium-derived factor (PEDF) levels, reduced the number of apoptotic cells as well as microglial infiltration in the retina. ${ }^{14}$ In Royal College of Surgeons (RCS) rats with an inherited mutation of MER proto-oncogene tyrosine kinase (MERTK) gene as a model of retinal degeneration, subretinal transplantation of iPSC-derived RPE cells significantly rescued visual function as measured by optokinetic tracking thresholds (OKT). None of the animals showed abnormal proliferation or teratoma formation; however, the graft was compromised in two animals due to inflammatory response. ${ }^{15}$ Interestingly, co-transplantation of RPCs and RPE cells derived from iPSCs was superior to transplanting individual cell types, it resulted in better visual response and preservation of ONL in a rat model of retinal degeneration. ${ }^{16}$

Further, in an animal model of RP, subretinally transplanted iPSC-derived CRX-expressing photoreceptor precursors engrafted at the inner nuclear layer (INL). The transplanted cells expressed the pan cone marker, Arrestin 3, indicating further maturation. ${ }^{17}$ In a preclinical study with rats and pigs, iPSCs obtained from AMD patient $\mathrm{CD}^{+} 4^{+}$cells, when differentiated into RPE cells, integrated and rescued the retinal degeneration. In this study, the authors found that, compared to suspension cells, ten times fewer RPE cells were required to achieve the same therapeutic effect when transplanted as a monolayer. Whereas RPE cells transplanted as cell suspension failed to integrate into the rat RPE layer, poly (lactic-coglycolic acid) (PLGA) based scaffold facilitated the integration of transplanted RPE patch into the rat Bruch's membrane. ${ }^{18}$ Stem cell-based therapies have also been explored as an option to treat retinal ischemic injuries with abnormal endothelial progenitor cells (EPCs) prevalent in diabetic patients. hiPSCderived endothelial cells alleviated oxygen-induced retinal injury in mouse models and reduced pathological vasoobliteration and neovascular tufts. ${ }^{19}$

\section{MSCs}

MSCs have been studied extensively for their potential in the treatment of several retinal disorders. Here, we have discussed some recent reports that utilized MSCs in the preclinical models of retinal degeneration. Human dental-pulp-derived MSCs (DP-MSCs) on intravitreal transplantation improved the retinal function in a rat model of retinal degeneration, ${ }^{20}$ and rat bone marrow-derived MSCs (BM-MSCs) rescued the ONL thickness by enhancing autophagy. ${ }^{21}$ Intravitreal injection of umbilical cord-derived MSCs (UC-MSCs), and the exosomes derived from UC-MSCs suppressed inflammatory response, retinal damage and improved the visual functions in a mouse model of retinal injury. ${ }^{22}$ Injection of mouse BM-MSCs or paracrine factors derived from BM-MSCs into the anterior ocular chamber induced proliferation of progenitor cells in the ciliary body and promoted ocular regeneration and repair in a glaucoma mouse model. ${ }^{23}$ Similarly, conditioned media (CM) from BM-MSCs containing the paracrine factors significantly reduced the intraocular pressure (IOP) and protected the host RGCs. ${ }^{24}$ Intravenous injection of UC-MSCs reduced diabetes-associated microvascular leakage in the retina by upregulating the expression of tight junction protein occludin. ${ }^{25}$ In a streptozotocin-induced diabetic mouse model of DR, intravitreal injection of adipose tissue-derived MSCs (AD-MSCs) increased intraocular levels of neurotrophic factors and prevented the loss of RGCs. ${ }^{26}$

Several studies have analyzed the potential of MSCderived factors, cells, and engineered MSCs to repair the damaged retina. Extracellular vesicles derived from human BM-MSCs significantly protected RGCs and prevented retinal nerve fiber layer thinning in a preclinical rat model of glaucoma. ${ }^{27}$ Injection of the stromal fraction of adipose tissue, which is enriched with pericytes, decreased vascular leakage, apoptosis and improved the "b" wave amplitude in a DR mouse model. ${ }^{28}$ Similar reductions in vascular leakage and improvements in visual acuity were observed when $\mathrm{CM}$ derived from human AD-MSCs were intravitreally injected in Ins $2^{\text {Akita }}$ mouse model of DR. ${ }^{29}$ Murine BM-MSCs genetically modified to produce neurotrophin-4 preserved the retinal bioelectrical activity in the injured retina and completely restored the laminated organization of the outer retina in an RP animal model. ${ }^{30}$ Similarly, BM-MSCs genetically engineered to express C-X-C chemokine receptor type $4,{ }^{31}$ or $\mathrm{PEDF}^{32}$ significantly reduced the retinal damage, reduced the 
level of pro-inflammatory cytokines, and restored the retinal structure and function in DR disease models. Interestingly, the administration of neural stem cells derived from UC-MSCs significantly improved the vision and survival of RGCs in diabetic rats. $^{33}$

\section{Clinical Trials with Stem Cells}

The encouraging results obtained from preclinical studies led to several clinical trials utilizing various stem cells and their derivatives.

\section{ESCs}

In an interim report on Phase $\mathrm{I} / \mathrm{IIa}$ clinical trial (NCT02286089) of 12 patients with advanced dry agerelated macular degeneration (AMD) and geographic atrophy (GA), Banin et al reported that hESC-derived RPE cells were well tolerated in patients when administered along with systemic immunosuppression before transplantation. Transplanted cells were detected in the subretinal space during long-term follow-up, and improvement in the RPE layers at the GA border was observed. ${ }^{34}$ Similarly, transplantation of hESC-derived RPE cells in AMD and Stargardt macular dystrophy (SMD) patients resulted in significant improvement in visual acuity without any abnormal proliferation. ${ }^{35,36}$ A recent update by Riemann et al on the fourth cohort of patients in phase I/IIa clinical trial (NCT02286089) reported visual improvements and alterations in the appearance of drusen in the treated patients. However, formation of epiretinal membranes (ERM) and retinal detachment was observed in some patients, which were successfully treated. ${ }^{37}$ In another clinical trial that tested the feasibility of utilizing ESC-derived RPE cells for retinal degeneration, da Cruz et al reported improved visual acuity in two patients with severe exudative AMD after subretinal transplantation. However, one of the patients experienced a reduction in photoreceptor function during the followup period, and the other patient had retinal detachment, which might or might not be related to the surgical procedure. ${ }^{38}$

\section{iPSCs}

Although ESC-derived RPE cells were functional and patients showed improvements in visual acuity, transplantation of ESCderived cells requires local or systemic immunosuppression. With the recent utilization of iPSCs to treat several disorders, Mandai et al reported interesting outcomes in the clinical trials with transplantation of iPSC-derived RPE cells. Autologous transplantation of iPSC-derived RPE cells in a patient with advanced neovascular AMD was well tolerated, and although the transplanted cell layer was intact, no improvement in the visual acuity was observed one year after the transplantation. ${ }^{39}$ A four-year follow-up on the same patient found that the transplanted cells supported the photoreceptors, and the visual acuity remained stable without anti-VEGF administration. ${ }^{40}$

\section{MSCs}

Mesenchymal stem cells (MSCs) and their derivatives were tested in numerous preclinical and clinical trials to treat retinal disorders. In Phase I clinical trial on 4 Asian patients with traumatic optic neuropathy, Sung et al found that sub-tenon transplantation of human placenta-derived MSCs (PD-MSCs) was safe without any adverse inflammatory or proliferative side effects. PD-MSCs had a protective effect on RGCs, rescued the expression of Tuj1 and GFAP, which was concurrent with improved visual acuity. ${ }^{41}$ In a non-randomized phase I clinical trial of 14 patients with RP, autologous BM-MSCs were transplanted intravitreally, and the improvement in visual function was assessed between 1 and 7 years. Immediately after transplantation, an increase in IOP was observed in all the patients that returned to baseline after $24 \mathrm{hr}$. All the participants showed improvement in BCVA (best-corrected visual acuity) a few months after transplantation; however, it returned to baseline within 12 months, and no further deterioration of the condition was observed. One of the participants developed a condition called osseous metaplasia in the ciliary body in the third year of follow-up, and another patient developed intraocular lens (IOL) subluxation in the fourth year. ${ }^{42}$

In Phase III clinical trial, Kahraman et al transplanted UCMSCs into the suprachoroidal area of $82 \mathrm{RP}$ patients involving 124 eyes. At a 6-month follow-up, $46 \%$ of eyes experienced an improvement in the vision, $42 \%$ of eyes remained stable, and $12 \%$ of eyes had the condition worsening, but none experienced adverse events. ${ }^{43}$ Similarly, in a phase I/II clinical trial of 32 RP patients, when UC-MSCs were administered intravenously, $90.6 \%$ of patients had improved visual acuity at $12-$ months follow-up. None of the patients experienced adverse effects; however, the average visual field sensitivity and flash visual evoked potential remained the same for all the patients following the transplantation. ${ }^{44}$ Several other clinical trials in RP patients reported improved visual acuity or significant changes when BM-MSCs were transplanted. ${ }^{45}$ In phase III clinical trial on 32 patients involving 34 eyes with RP, subtenon transplantation of Wharton's jelly-derived MSCs (WJMSCs) significantly improved BCVA, visual field, and outer retinal thickness during a 6-month follow-up. The transplanted cells did not induce any adverse side-effects with the study still on-going. ${ }^{46}$ In a clinical trial involving patients with proliferative DR (PDR) and non-proliferative DR (NPDR), intravenous 
transplantation of autologous BM-MSCs significantly improved the BCVA at 3- and 6-months follow-up period in the NPDR group but not the PDR patients. Injection of BMMSCs was followed by intravenous administration of dexamethasone sodium, and the transplantation did not cause any adverse immune reactions systemically or at the ocular site. ${ }^{47}$ In another clinical trial involving two patients with advanced glaucoma, with intravitreal injection of autologous BM-MSCs, none of the patients showed improvements in visual acuity or visual field during a 12-month follow-up, but one of the patients experienced retinal detachment two-weeks after the treatment initiation. ${ }^{48}$

In addition to transplantation of stem cells cultured ex vivo before transplantation, a few clinical trials examined the direct injection of stem cell population isolated from bone marrow or adipose tissue. Recently, Wiącek et al reported intravitreal transplantation of autologous bone marrow-derived lineagenegative (BM lin ${ }^{-}$) cells in RP patients with the disease incidence ranging from few years to more than 10 years. ${ }^{49}$ A significant improvement in the BCVA and BCDVA (bestcorrected distance visual acuity) was reported by the patients at the 12-months follow-up period, and improvement in the visual parameters was more pronounced in patients who had symptoms for less than 10 years and maintained functional foveal cones. The study also reported retinal detachment in three cases, with two cases requiring surgery to achieve complete retinal attachment. ${ }^{49}$ In two separate clinical trials, Limoli et al transplanted autologous adipose-derived stem cells (ADSCs) from the stromal vascular fraction of the adipose tissue along with platelets obtained from platelet-rich plasma and adipose stromal cells of the orbital fat in the subscleral space. The study reported an improvement in visual performance with no adverse effects. ${ }^{50,51}$ In an experimental clinical trial, when patients with refractory macular holes were injected with either UC-MSCs or exosomes derived from UC-MSCs, improvement in BCVA and closure of macular holes in six out of seven patients was observed. No adverse reaction was observed, except one patient who showed inflammatory response due to the high dose of exosomes. ${ }^{52}$

\section{Challenges with Stem Cells Therapy}

In addition to the protective and regenerative effect identified with the transplantation of stem cells for retinal diseases, several clinical and preclinical studies have also reported transplantation-related adverse effects. For example, in a rat model of anterior ischemic optic neuropathy, intravitreal transplantation of WJ-MSCs induced retinal damage and severe inflammation, accompanied by macrophage infiltration.
However, intravitreal injection of CM from MSCs inhibited apoptosis of RGCs, reduced inflammation by inhibiting microglia activation and macrophage infiltration. ${ }^{53}$ In the Pde6brd 1 mice model of autosomal recessive form of RP, subretinally transplanted iPSC-derived $\mathrm{CRX}+$ photoreceptor precursor cells engrafted into the retina and differentiated into cones, leading to improved visual behavior scores. However, an "unconventional" light response was observed after transplantation due to novel synapse formation between cones and RGCs. $^{54}$

In a case study of a patient with RP associated with Usher Syndrome, intravitreal transplantation of autologous adipose tissue-derived stem cells induced neovascular glaucoma, gradual vision loss, and retinal detachment. ${ }^{55}$ Several clinical trials have reported adverse effects of retinal detachment and vision loss following stem cell transplantations. ${ }^{56}$ Transplantation of hESCs, autologous BM-MSCs, resulted in retinal detachment in AMD patients ${ }^{37,38}$ and glaucoma, ${ }^{48}$ and RP patients ${ }^{49}$ respectively. Some of them required surgical interventions to facilitate retinal attachment. Other adverse effects include inflammatory response due to the injections of the cells ${ }^{15}$ or its factors. ${ }^{42}$ These adverse effects might be due to the invasive nature of intravitreal transplantation, and thus further research on the optimal delivery route for each disease type is required.

As mentioned earlier, due considerations should be given to the transplantation route and immune suppression during transplantation. Hu et al found that transplantation of autologous rat ASCs in the vitreous chamber reduced apoptosis and distortion of retinal structure; however, it induced retinal folding and failed to improve the ERG "b" wave response. In contrast, the subretinal transplantation of ASCs significantly improved the "b" wave response and was deemed a more suitable transplantation site for stem cells. ${ }^{57}$ Several studies have noted positive integration of the transplanted cells in the retinal layer and improved visual acuity in the short-term follow-up; however, a long-term improvement in the vision and quality of life has not been studied. In addition, several studies also lack long-term follow-up regarding the safety and the functioning of the transplanted cells.

Recent studies have highlighted another caveat in assessing the integration of transplanted photoreceptor cells in the host retina. Several studies have used fluorescent reporters to establish the integration of transplants forming novel synaptic connections in the host retina. ${ }^{58,59}$ However, bidirectional material transfer of cytoplasmic and nuclear proteins between transplanted cells and host photoreceptors has been shown to account for most of such integration instances. ${ }^{59}$ Therefore, 
caution should be exercised while interpreting the integration of the transplanted stem cells in the host retina.

\section{Conclusions and Future Directions}

Given the positive outcomes obtained from several preclinical and clinical studies, stem cell therapy remains an excellent option for the treatment of retinal degeneration. In addition to transplanting stem cells and their derivatives, future therapeutic options can include cotransplantation of two or more types of cells to achieve a better clinical benefit. ${ }^{16,60}$ Further, stem cells can be modified to overexpress retinal regenerative factors, ${ }^{31,32,61}$ and utilization of scaffolds to culture and transplant the stem cells and their derivations might improve their clinical benefits. ${ }^{62,63}$

However, there is a lack of consensus on the route of administration, method of evaluation of outcome, source of stem cells, and the long-term effect of stem cell transplantations. Donor-based variations in the functioning of RPE cells derived from iPSCs were observed, ${ }^{18}$ which should be considered before transplantation. Agerelated and niche-based changes in the function of MSCs have been well documented ${ }^{64}$ and should be given due consideration while utilizing them for clinical use. Thus, cell-line banks with modified or unmodified stem cells tested to have the best clinical outcome can be established to overcome the donor-based and culture-based heterogeneity. Moreover, standard culture conditions should be established for expanding the stem cells to avoid variations incorporated due to culture conditions and in vitro aging. Although RPE cells, RPCs derived from hESCs, iPSCs but not undifferentiated cells were injected during therapy, the possibility of tumor formation exists from the residual undifferentiated cells. To date, data on the long-term safety of cells derived from hESCs and iPSCs in terms of teratoma formation after transplantation is not available; thus, MSCs and their derivatives might be more suitable candidates for the treatment of retinal degeneration.

\section{Acknowledgments}

AS was supported by the Ministry of Education (MoE), Govt. of India. This study was partially supported by the Indian Institute of Technology Guwahati (IITG).

\section{Disclosure}

The authors declare that they have no conflicts of interest.

\section{References}

1. Shen Y. Stem cell therapies for retinal diseases: from bench to bedside. J Mol Med. 2020;98(10):1347-1368. doi:10.1007/s00109020-01960-5

2. Eandi CM, Alovisi C, De Sanctis U, Grignolo FM. Treatment for neovascular age related macular degeneration: the state of the art. Eur J Pharmacol. 2016;787:78-83. doi:10.1016/j.ejphar.2016.03.002

3. Singh SR, Fung AT, Fraser-Bell S, et al. One-year outcomes of anti-vascular endothelial growth factor therapy in peripapillary choroidal neovascularisation. $\mathrm{Br} J$ Ophthalmol. 2020;104(5):678-683. doi:10.1136/bjophthalmol-2019-314542

4. Ran X, Cai WJ, Huang XF, et al. 'RetinoGenetics': a comprehensive mutation database for genes related to inherited retinal degeneration. Database. 2014;2014:bau071-bau071. doi:10.1093/database/bau047

5. Prado DA, Acosta-Acero M, Maldonado RS. Gene therapy beyond luxturna: a new horizon of the treatment for inherited retinal disease. Curr Opin Ophthalmol. 2020;31(3):147-154. doi:10.1097/ icu. 0000000000000660

6. da Cruz L, Dorn JD, Humayun MS, et al. Five-year safety and performance results from the argus II retinal prosthesis system clinical trial. Ophthalmology. 2016;123(10):2248-2254. doi:10.1016/j. ophtha.2016.06.049

7. Krick TW, Bressler NM. Recent clinically relevant highlights from the diabetic retinopathy clinical research network. Curr Opin Ophthalmol. 2018;29(3):199-205. doi:10.1097/icu.0000000000000472

8. Petrus-Reurer S, Kumar P, Padrell Sánchez S, et al. Preclinical safety studies of human embryonic stem cell-derived retinal pigment epithelial cells for the treatment of age-related macular degeneration. Stem Cells Transl Med. 2020;9(8):936-953. doi:10.1002/sctm.19-0396

9. Mazzilli JL, Snook JD, Simmons K, et al. A preclinical safety study of human embryonic stem cell-derived retinal pigment epithelial cells for macular degeneration. J Ocular Pharmacol Ther. 2020;36 (1):65-69. doi:10.1089/jop.2019.0039

10. Wang ST, Chen LL, Zhang P, et al. Transplantation of retinal progenitor cells from optic cup-like structures differentiated from human embryonic stem cells in vitro and in vivo generation of retinal ganglion-like cells. Stem Cells Dev. 2019;28(4):258-267. doi: $10.1089 / \mathrm{scd} .2018 .0076$

11. Wang Z, Gao F, Zhang M, et al. Intravitreal injection of human retinal progenitor cells for treatment of retinal degeneration. Med Sci Monitor. 2020;26:e921184-e921184. doi:10.12659/MSM.921184

12. Aboualizadeh E, Phillips MJ, McGregor JE, et al. Imaging transplanted photoreceptors in living nonhuman primates with single-cell resolution. Stem Cell Rep. 2020;15(2):482-497. doi:10.1016/j. stemcr.2020.06.019

13. Tu HY, Watanabe T, Shirai H, et al. Medium- to long-term survival and functional examination of human iPSC-derived retinas in rat and primate models of retinal degeneration. EBioMedicine. 2019;39:562-574. doi:10.1016/j.ebiom.2018.11.028

14. Zhu D, Xie M, Gademann F, et al. Protective effects of human iPS-derived retinal pigmented epithelial cells on retinal degenerative disease. Stem Cell Res Ther. 2020;11(1):98. doi:10.1186/s13287-020-01608-8

15. Surendran H, Nandakumar S, Reddy KV, et al. Transplantation of retinal pigment epithelium and photoreceptors generated concomitantly via small molecule-mediated differentiation rescues visual function in rodent models of retinal degeneration. Stem Cell Res Ther. 2021;12(1):70. doi:10.1186/s13287-021-02134-x

16. Salas A, Duarri A, Fontrodona L, et al. Cell therapy with hiPSC-derived RPE cells and RPCs prevents visual function loss in a rat model of retinal degeneration. Mol Ther Methods Clin Develop. 2021;20:688-702. doi:10.1016/j.omtm.2021.02.006

17. Collin J, Zerti D, Queen R, et al. CRX expression in pluripotent stem cell-derived photoreceptors marks a transplantable subpopulation of early cones. Stem Cells (Dayton, Ohio). 2019;37(5):609-622. doi:10.1002/stem.2974 
18. Sharma R, Khristov V, Rising A, et al. Clinical-grade stem cell-derived retinal pigment epithelium patch rescues retinal degeneration in rodents and pigs. Sci Transl Med. 2019;11(475):eaat5580. doi:10.1126/scitranslmed.aat5580

19. Cho H, Macklin BL, Lin YY, et al. iPSC-derived endothelial cell response to hypoxia via SDF1a/CXCR4 axis facilitates incorporation to revascularize ischemic retina. JCI Insight. 2020;5(6). doi:10.1172/ jci.insight. 131828

20. Alsaeedi HA, Koh AE, Lam C, et al. Dental pulp stem cells therapy overcome photoreceptor cell death and protects the retina in a rat model of sodium iodate-induced retinal degeneration. $J$ Photochem Photobiol B. 2019;198:111561. doi:10.1016/j.jphotobiol.2019.111561

21. Liu X, Xie J, Yang L, et al. Bone marrow mesenchymal stem cells enhance autophagy and help protect cells under hypoxic and retinal detachment conditions. J Cell Mol Med. 2020;24(6):3346-3358. doi: $10.1111 / \mathrm{jcmm} .15008$

22. Yu B, Shao H, Su C, et al. Exosomes derived from MSCs ameliorate retinal laser injury partially by inhibition of MCP-1. Sci Rep 2016;6:34562. doi:10.1038/srep34562

23. Manuguerra-Gagné R, Boulos PR, Ammar A, et al. Transplantation of mesenchymal stem cells promotes tissue regeneration in a glaucoma model through laser-induced paracrine factor secretion and progenitor cell recruitment. Stem Cells (Dayton, Ohio). 2013;31 (6):1136-1148. doi:10.1002/stem.1364

24. Roubeix C, Godefroy D, Mias C, et al. Intraocular pressure reduction and neuroprotection conferred by bone marrow-derived mesenchymal stem cells in an animal model of glaucoma. Stem Cell Res Ther. 2015;6(1):177. doi:10.1186/s13287-015-0168-0

25. Yu C, Yang K, Meng X, Cao B, Wang F. Downregulation of long noncoding RNA MIAT in the retina of diabetic rats with tail-vein injection of human umbilical-cord mesenchymal stem cells. Int $J$ Med Sci. 2020;17(5):591-598. doi:10.7150/ijms.38078

26. Ezquer M, Urzua CA, Montecino S, Leal K, Conget P, Ezquer F. Intravitreal administration of multipotent mesenchymal stromal cells triggers a cytoprotective microenvironment in the retina of diabetic mice. Stem Cell Res Ther. 2016;7:42. doi:10.1186/s13287-016-0299-y

27. Mead B, Amaral J, Tomarev S. Mesenchymal stem cell-derived small extracellular vesicles promote neuroprotection in rodent models of glaucoma. Invest Ophthalmol Vis Sci. 2018;59(2):702-714. doi:10.1167/iovs.17-22855

28. Rajashekhar G, Ramadan A, Abburi C, et al. Regenerative therapeutic potential of adipose stromal cells in early stage diabetic retinopathy. PLoS One. 2014;9(1):e84671. doi:10.1371/journal. pone. 0084671

29. Elshaer SL, Evans W, Pentecost M, et al. Adipose stem cells and their paracrine factors are therapeutic for early retinal complications of diabetes in the Ins2(Akita) mouse. Stem Cell Res Ther. 2018;9 (1):322. doi:10.1186/s13287-018-1059-y

30. Machalińska A, Kawa M, Pius-Sadowska E, et al. Long-term neuroprotective effects of NT-4-engineered mesenchymal stem cells injected intravitreally in a mouse model of acute retinal injury. Invest Ophthalmol Vis Sci. 2013;54(13):8292-8305. doi:10.1167/ iovs.13-12221

31. Wang J, Zhang W, He GH, Wu B, Chen S. Transfection with CXCR4 potentiates homing of mesenchymal stem cells in vitro and therapy of diabetic retinopathy in vivo. Int $J$ Ophthalmol. 2018;11(5):766-772. doi:10.18240/ijo.2018.05.08

32. Kim JY, Park S, Park SH, et al. Overexpression of pigment epithelium-derived factor in placenta-derived mesenchymal stem cells promotes mitochondrial biogenesis in retinal cells. Lab Invest. 2021;101(1):51-69. doi:10.1038/s41374-020-0470-z

33. Zhang W, Wang Y, Kong J, Dong M, Duan H, Chen S. Therapeutic efficacy of neural stem cells originating from umbilical cord-derived mesenchymal stem cells in diabetic retinopathy. Sci Rep. 2017;7 (1):408. doi:10.1038/s41598-017-00298-2
34. Banin E, Barak A, Boyer DS, et al. Phase I/IIa Clinical Trial of Human Embryonic Stem Cell (hESC)-derived retinal pigmented epithelium (RPE, OpRegen) transplantation in advanced dry form Age-Related Macular Degeneration (AMD): interim results. Invest Ophthalmol Vis Sci. 2019;60(9):6402.

35. Schwartz SD, Regillo CD, Lam BL, et al. Human embryonic stem cell-derived retinal pigment epithelium in patients with age-related macular degeneration and Stargardt's macular dystrophy: follow-up of two open-label Phase 1/2 studies. Lancet (London, England). 2015;385(9967):509-516. doi:10.1016/s0140-6736(14)61376-3

36. Song WK, Park KM, Kim HJ, et al. Treatment of macular degeneration using embryonic stem cell-derived retinal pigment epithelium: preliminary results in Asian patients. Stem Cell Rep. 2015;4 (5):860-872. doi:10.1016/j.stemcr.2015.04.005

37. Riemann CD, Banin E, Barak A, et al. Phase I/IIa clinical trial of Human Embryonic Stem Cell (hESC)-derived Retinal Pigmented Epithelium (RPE, OpRegen) transplantation in advanced dry form Age-Related Macular Degeneration (AMD): interim results. Invest Ophthalmol Vis Sci. 2020;61(7):865.

38. da Cruz L, Fynes K, Georgiadis O, et al. Phase 1 clinical study of an embryonic stem cell-derived retinal pigment epithelium patch in age-related macular degeneration. Nat Biotechnol. 2018;36 (4):328-337. doi:10.1038/nbt.4114

39. Mandai M, Watanabe A, Kurimoto $\mathrm{Y}$, et al. Autologous induced stem-cell-derived retinal cells for macular degeneration. $N$ Engl J Med. 2017;376(11):1038-1046. doi:10.1056/NEJMoa1608368

40. Takagi S, Mandai M, Gocho K, et al. Evaluation of transplanted autologous induced pluripotent stem cell-derived retinal pigment epithelium in exudative age-related macular degeneration. Ophthalmol Retina. 2019;3 (10):850-859. doi:10.1016/j.oret.2019.04.021

41. Sung Y, Lee SM, Park M, et al. Treatment of traumatic optic neuropathy using human placenta-derived mesenchymal stem cells in Asian patients. Regen Med. 2020;15(10):2163-2179. doi:10.2217/ rme-2020-0044

42. Tuekprakhon A, Sangkitporn S, Trinavarat A, et al. Intravitreal autologous mesenchymal stem cell transplantation: a non-randomized phase I clinical trial in patients with retinitis pigmentosa. Stem Cell Res Ther. 2021;12(1):52. doi:10.1186/s13287-020-02122-7

43. Kahraman NS, Oner A. Umbilical cord derived mesenchymal stem cell implantation in retinitis pigmentosa: a 6-month follow-up results of a Phase 3 trial. Int $J$ Ophthalmol. 2020;13(9):1423-1429. doi:10.18240/ijo.2020.09.14

44. Zhao T, Liang Q, Meng X, et al. Intravenous infusion of umbilical cord mesenchymal stem cells maintains and partially improves visual function in patients with advanced retinitis pigmentosa. Stem Cells Dev. 2020;29(16):1029-1037. doi:10.1089/scd.2020.0037

45. Adak S, Magdalene D, Deshmukh S, Das D, Jaganathan BG. A review on mesenchymal stem cells for treatment of retinal diseases. Stem Cell Rev Rep. 2021;1-20. doi:10.1007/s12015-02010090-x

46. Özmert E, Arslan U. Management of retinitis pigmentosa by Wharton's jelly derived mesenchymal stem cells: preliminary clinical results. Stem Cell Res Ther. 2020;11(1):25. doi:10.1186/s13287-0201549-6

47. Gu X, Yu X, Zhao C, et al. Efficacy and safety of autologous bone marrow mesenchymal stem cell transplantation in patients with diabetic retinopathy. Cell Physiol Biochem. 2018;49(1):40-52. doi: $10.1159 / 000492838$

48. Vilela CAP, Messias A, Calado RT, et al. Retinal function after intravitreal injection of autologous bone marrow-derived mesenchymal stromal cells in advanced glaucoma. Doc Ophthalmol. 2021;143 (1):33-38. doi:10.1007/s10633-021-09817-z

49. Wiącek MP, Gosławski W, Grabowicz A, et al. Long-term effects of adjuvant intravitreal treatment with autologous bone marrow-derived lineage-negative cells in retinitis pigmentosa. Stem Cells Int. 2021;2021:6631921. doi:10.1155/2021/6631921 
50. Limoli PG, Limoli C, Vingolo EM, Scalinci SZ, Nebbioso M. Cell surgery and growth factors in dry age-related macular degeneration: visual prognosis and morphological study. Oncotarget. 2016;7 (30):46913-46923. doi:10.18632/oncotarget.10442

51. Limoli PG, Vingolo EM, Morales MU, Nebbioso M, Limoli C. Preliminary study on electrophysiological changes after cellular autograft in age-related macular degeneration. Medicine. 2014;93(29): e355. doi: $10.1097 / \mathrm{md} .0000000000000355$

52. Zhang X, Liu J, Yu B, Ma F, Ren X, Li X. Effects of mesenchymal stem cells and their exosomes on the healing of large and refractory macular holes. Graefe's Arch Clin Exp Ophthalmol/Albrecht von Graefes Archiv fur klinische und experimentelle Ophthalmologie. 2018;256(11):2041-2052. doi:10.1007/s00417-018-4097-3

53. Wen YT, Ho YC, Lee YC, Ding DC, Liu PK, Tsai RK. The benefits and hazards of intravitreal Mesenchymal Stem Cell (MSC) based-therapies in the experimental ischemic optic neuropathy. Int J Mol Sci. 2021;22(4):2117. doi:10.3390/ijms22042117

54. Zerti D, Hilgen G, Dorgau B, et al. Transplanted pluripotent stem cell-derived photoreceptor precursors elicit conventional and unusual light responses in mice with advanced retinal degeneration. Stem Cells (Dayton, Ohio). 2021;39(7):882-896. doi:10.1002/stem.3365

55. Khine KT, Albini TA, Lee RK. Chronic retinal detachment and neovascular glaucoma after intravitreal stem cell injection for Usher Syndrome. Am J Ophthalmol Case Rep. 2020;18:100647. doi:10.1016/j.ajoc.2020.100647

56. Rong AJ, Lam BL, Ansari ZA, Albini TA. Vision loss secondary to autologous adipose stem cell injections: a rising problem. JAMA Ophthalmol. 2018;136(1):97-99. doi:10.1001/jamaophthalm ol.2017.5453

57. Hu C, La H, Wei X, et al. Transplantation site affects the outcomes of adipose-derived stem cell-based therapy for retinal degeneration. Stem Cells Int. 2020;2020:9625798. doi:10.1155/2020/9625798
58. Bartsch U, Oriyakhel W, Kenna PF, et al. Retinal cells integrate into the outer nuclear layer and differentiate into mature photoreceptors after subretinal transplantation into adult mice. Exp Eye Res. 2008;86 (4):691-700. doi:10.1016/j.exer.2008.01.018

59. Gasparini SJ, Llonch S, Borsch O, Ader M. Transplantation of photoreceptors into the degenerative retina: current state and future perspectives. Prog Retin Eye Res. 2019;69:1-37. doi:10.1016/j. preteyeres.2018.11.001

60. Pan T, Shen H, Yuan S, et al. Combined transplantation with human mesenchymal stem cells improves retinal rescue effect of human fetal RPE cells in retinal degeneration mouse model. Invest Ophthalmol Vis Sci. 2020;61(8):9. doi:10.1167/iovs.61.8.9

61. Huang L, You J, Yao Y, Xie M. Interleukin-13 gene modification enhances grafted mesenchymal stem cells survival after subretinal transplantation. Cell Mol Neurobiol. 2020;40(5):725-735. doi:10.1007/s10571-019-00768-3

62. Colombe Dromel P, Singh D, Alexander-Katz A, Kurisawa M, Spector M, Young M. Injectable gelatin hydroxyphenyl propionic acid hydrogel protects human retinal progenitor cells (hRPCs) from shear stress applied during small-bore needle injection. Appl Mater Today. 2020;19:100602. doi:10.1016/j.apmt.2020.100602

63. Tian Y, Davis R, Zonca MR Jr, Stern JH, Temple S, Xie Y. Screening and optimization of potential injection vehicles for storage of retinal pigment epithelial stem cell before transplantation. J Tissue Eng Regen Med. 2019;13(1):76-86. doi:10.1002/term.2770

64. Costa LA, Eiro N, Fraile M, et al. Functional heterogeneity of mesenchymal stem cells from natural niches to culture conditions: implications for further clinical uses. Cell Mol Life Sci. 2021;78 (2):447-467. doi:10.1007/s00018-020-03600-0

\section{Publish your work in this journal}

Biologics: Targets and Therapy is an international, peer-reviewed journal focusing on the patho-physiological rationale for and clinical application of Biologic agents in the management of autoimmune diseases, cancers or other pathologies where a molecular target can be identified. This journal is indexed on PubMed Central, CAS, EMBase,
Scopus and the Elsevier Bibliographic databases. The manuscript management system is completely online and includes a very quick and fair peer-review system, which is all easy to use. Visit http://www.dovepress.com/testimonials.php to read real quotes from published authors. 